Review Article

\title{
Diagnostic yield of capsule endoscopy versus magnetic resonance enterography and small bowel contrast ultrasound in the evaluation of small bowel Crohn's disease: Systematic review and meta-analysis
}

\author{
Uri Kopylov $^{\mathrm{a}, *}$, Diana E. Yung ${ }^{\mathrm{b}}$, Tal Engel ${ }^{\mathrm{a}}$, Sanju Vijayan ${ }^{\mathrm{b}}$, Ofir Har-Noy ${ }^{\mathrm{a}}$, Lior Katz ${ }^{\mathrm{a}}$, \\ Salvatore Oliva ${ }^{\mathrm{c}}$, Tomer Avni ${ }^{\mathrm{d}}$, Robert Battat ${ }^{\mathrm{e}}$, Rami Eliakim ${ }^{\mathrm{a}}$, Shomron Ben-Horin ${ }^{\mathrm{a}}$, \\ Anastasios Koulaouzidis ${ }^{\text {b }}$ \\ a Gastroenterology Department, Sheba Medical Center, Ramat-Gan, and Sackler School of Medicine, Tel-Aviv University, Israel \\ ${ }^{\mathrm{b}}$ Centre for Liver E' Digestive Disorders, The Royal Infirmary of Edinburgh, Edinburgh, United Kingdom \\ c Pediatric Gastroenterology and Liver Unit, Sapienza University of Rome, Italy \\ ${ }^{\mathrm{d}}$ Department of Medicine E, Davidoff Cancer Center, Rabin Medical Center, Beilinson Hospital, Petah-Tikva, and Sackler School of Medicine, Tel-Aviv \\ University, Israel \\ e McGill University Health Center, McGill University, Montreal, QC, Canada
}

\section{A R T I C L E I N F O}

\section{Article history:}

Received 13 February 2017

Received in revised form 7 April 2017

Accepted 18 April 2017

Available online 27 April 2017

\section{Keywords:}

Capsule endoscopy

Magnetic resonance enterography

Small bowel ultrasound

\begin{abstract}
A B S T R A C T
Background and aims: Capsule endoscopy (CE), magnetic resonance enterography (MRE) and small bowel (SB) intestinal contrast ultrasound (SICUS) are the modalities of choice for SB evaluation. This study aimed to compare the diagnostic yield (DY) of CE to MRE and SICUS in detection and monitoring of SB CD through meta-analysis of the available literature.

Methods: We performed a systematic literature search for trials comparing the accuracy of CE, MRE and SICUS for detection of active SB inflammation in patients with suspected and/or established CD. Only prospective studies comparing CE with another additional diagnostic modality were included in the final analysis. Pooled odds ratios (ORs) for the DY of the three modalities were calculated.

Results: A total of 112 studies were retrieved; following selection, 13 studies were eligible for analysis. The DY of CE for detection of active SB CD was similar to that of MRE (10 studies, 400 patients, OR 1.17; 95\% CI 0.83-1.67) and SICUS (5 studies, 142 patients, OR 0.88; 95\% CI 0.51-1.53). The outcomes were similar for the subgroups of suspected versus established CD and adult versus pediatric patients. CE was superior to MRE for proximal SB CD ( 7 studies, 251 patients, OR 2.79; 95\% CI 1.2-6.48); the difference vs SICUS was not significant.

Conclusion: CE, MRE and SICUS have similar DY for detection of SB CD in both suspected and established CD. CE is superior to MRE for detection of proximal SB disease, however the risk of capsule retention should be considered.
\end{abstract}

(c) 2017 Editrice Gastroenterologica Italiana S.r.l. Published by Elsevier Ltd. All rights reserved.

\section{Introduction}

Crohn's disease $(\mathrm{CD})$ may involve any part of the gastrointestinal (GI) tract. The small bowel (SB) is most commonly affected in at least $70 \%$ of patients, while in $30 \%$ CD is limited to the SB. The involved SB segments are frequently proximal to the terminal ileum and thus inaccessible to conventional endoscopic evaluation [1]. Nowadays, several modalities are available for SB

* Corresponding author at: Gastroenterology Department, Sheba Medical Center, Emek HaEla St 1, Ramat Gan, Israel.

E-mail address: ukopylov@gmail.com (U. Kopylov). assessment e.g. capsule endoscopy (CE), computer tomography (CT)-enterography, magnetic resonance enterography (MRE) (with several different techniques and protocols such as MR enteroclysis and diffusion-weighted MRE), and small bowel ultrasound (including SB contrast-enhanced ultrasound (SICUS)). Nevertheless, each of the aforementioned modalities has its own strengths and limitations.

Due to concerns of repeated radiation exposure, MRE is preferred to CTE for routine elective assessment of the SB [2]. A recent meta-analysis by Dionisio et al. demonstrated the superior diagnostic yield (DY) of CE to CTE and SB follow-through (SBFT), while there was no significant difference between the accuracy of MRE and CE [3]. Since then, several new studies incorporating novel diagnostic 


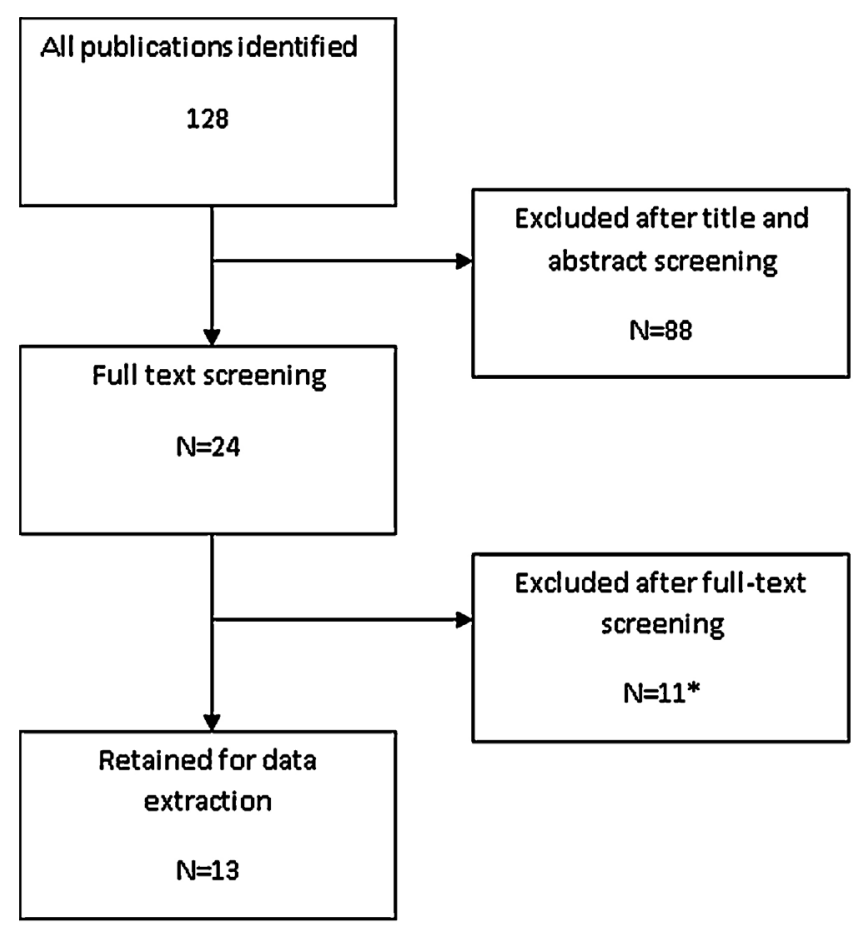

Fig. 1. Flow chart detailing process of study selection.

techniques have been published. This study aimed to compare the DY of CE, MRE and SICUS in detection and monitoring of SB CD by performing a systematic review and meta-analysis of the current literature.

\section{Methods}

A comprehensive literature search was conducted in July 2016 using the PubMed and Embase databases (January 2000-July 2016). In order to capture as many citations as possible, a broad search strategy was employed by combining the terms "capsule endoscopy", "magnetic resonance", "ultrasound", "small bowel" and "Crohn's disease" OR "inflammatory bowel disease" (as keywords and MeSH headings). References of the included studies and relevant reviews were scanned for additional suitable publications (Fig. 1).

For a study to be included in this meta-analysis, the following criteria were considered necessary:

a) Prospective studies

b) Studies comparing CE to either MRE or SICUS or both.

c) Published in full form in peer-reviewed literature in English

d) Including at least 10 patients undergoing CE

e) Studies that did not have CE as one of the diagnostic modalities were excluded.

Data extraction and quality control were performed independently by 4 reviewers (SV, TE, LK, DY). Two expert reviewers (IK, AK) were involved if there was any uncertainty about the data. Where additional data were required, primary (first and/or senior) authors of the corresponding manuscript(s) were contacted by email with the relevant questions.

\subsection{Outcome measures}

- For detection of lesions, we used number of findings detected by each modality as per specific criteria. As there is no established gold $=$ standard modality for detection of small bowel inflamma- tion, we considered all detected findings on either modality as positive. We calculated the DY both as "per protocol" (number of positive examinations out of total number of patients tested) and "intention to treat" (ITT) (number of positive examinations out of total number of patients referred), for patients excluded from CE due to SB strictures or other contraindications but evaluated by an alternative modality.

- The primary analysis was DY of CE vS MRE or SICUS for detection of SB disease in suspected and/or established CD. Secondary subgroup analyses were: suspected/established CD, pediatric/adult and analysis of only studies with a low risk of bias. Diagnostic yield was calculated separately for the proximal (jejunum/proximal ileum) and distal (terminal/distal ileum) SB when possible. Any colonic data was excluded from analysis.

\subsection{Statistical analysis}

Data on the DY of CE were extracted, pooled, and analyzed. Pooled results with corresponding odds ratios (OR) and 95\% confidence intervals (CI) were derived using the fixed effects model (Mantel-Haenszel method) unless significant heterogeneity was detected, in which case, a random-effects model (DerSimonian-Laird) was used. We used the Q statistic of $\chi^{2}$ test and $I^{2}$ to estimate the heterogeneity of individual studies contributing to the pooled estimate. $P<0.05$ suggests the presence of heterogeneity beyond what could be expected by chance alone. $I^{2}$ describes the percentage of total variation across studies attributed to heterogeneity rather than chance. An $I^{2}$ of $20-50 \%$ suggests moderate and $I^{2}>50 \%$ high heterogeneity. Forest plots were constructed for visual display of individual studies and pooled results [4]. The Fstatistic was used to determine significance in repeated measures ANOVA. $P<0.05$ for the F-statistic was considered statistically significant [5]. Planned sensitivity analyses included pediatric studies and studies with low risk of bias. Statistical analysis was performed by using the Metan package of STATA version 12.1 (StataCorp, College Station, Tex).

\subsection{Assessment of study bias}

Methodological quality and potential bias of the included studies was evaluated by using the QUality Assessment of Diagnostic Accuracy Studies (QUADAS) 2 scale [6].

\section{Results}

The initial search yielded 112 publications. After review of titles and abstracts, 88 papers were excluded for the following reasons: reviews/editorials/letters/opinion papers $(n=78)$, case reports $(n=4)$, not in English language $(n=1)$, not using CE $(n=5)$. Twenty four papers proceeded to full-text review, following which 11 papers were excluded for the following reasons: same patient cohort as another included study [7-10] $(n=4)$, retrospective [11] $(\mathrm{n}=1)$, mixed indications [12] $(\mathrm{n}=1)$, data presentation did not allow for comparison between modalities [13] $(n=1)$, inadequate sample size [14] ( $\mathrm{n}=1)$; cost-effectiveness study [15] $(\mathrm{n}=1)$; studies using patency and not diagnostic CE $[16,17](n=2)$.

\subsection{Characteristics of the included studies}

Thirteen studies (500 patients) were included (Table 1$)$. All studies were of European origin: (1- Denmark [18] $(n=1)$, the Netherlands [19] $(n=1)$, Israel [20] $(n=1)$, Germany [21-24] $(n=4)$, Italy $[25-30](n=6))$. Three studies involved pediatric patients $[25,27,28]$, while the rest evaluated adult patients only. Two studies included only patients with suspected CD [27,29], five studies 
summary of included studies in this metaanalysis.

\begin{tabular}{|c|c|c|c|c|c|c|c|c|c|c|c|c|}
\hline Authors, reference & Country & $\begin{array}{l}\text { Paediatric/ } \\
\text { Adult }\end{array}$ & $\begin{array}{l}\text { Suspected/ } \\
\text { established } \\
\text { CD }\end{array}$ & $\begin{array}{l}\text { Modality } \\
\text { compared } \\
\text { with CE }\end{array}$ & Total patients & $\begin{array}{l}\text { CE results } \\
\text { (+ve/total) }\end{array}$ & CE retentions & MRE technique & $\begin{array}{l}\text { MRE diagnostic } \\
\text { criteria }\end{array}$ & $\begin{array}{l}\text { MRE results } \\
\text { (+ve/total) }\end{array}$ & $\begin{array}{l}\text { US diagnostic } \\
\text { criteria }\end{array}$ & $\begin{array}{l}\text { US } \\
\text { results } \\
\text { (+ve/total) }\end{array}$ \\
\hline Albert et al. [24] & Germany & Adult & Both & MRE & $\begin{array}{l}52 \\
\text { Est: } 27 \\
\text { Susp: } 25\end{array}$ & $\begin{array}{l}25 / 27 \\
\text { Est: } 13 / 14 \\
\text { Susp: } 12 / 13\end{array}$ & 0 & Enteroclysis & $\begin{array}{l}>4 \mathrm{~mm} \mathrm{SB} \text { wall } \\
\text { thickening } \& \\
\text { enhancement }\end{array}$ & $\begin{array}{l}32 / 52 \\
\text { Est: } 22 / 27 \\
\text { Susp: } 10 / 25\end{array}$ & - & - \\
\hline Gölder et al. [22] & Germany & Adult & Established & MRE & 16 & $11 / 15$ & 0 & Enteroclysis & $\begin{array}{l}\text { SB wall thickening, } \\
\text { mesenteric } \\
\text { injection, enhanced } \\
\text { LNs }\end{array}$ & $9 / 15$ & - & - \\
\hline Biancone et al. [26] & Italy & Adult & Established & US & 22 & $16 / 17$ & 0 & - & - & - & $\begin{array}{l}>3 \mathrm{~mm} \text { SB wall } \\
\text { thickening, } \\
\text { "stiff loop", SB } \\
\text { dilation } \\
>2.5 \mathrm{~cm}, \\
\text { stricture }<1 \mathrm{~cm} \text {, } \\
\text { fistulas, } \\
\text { abscesses, } \\
\text { mesenteric } \\
\text { enlarge- } \\
\text { ment/masses }\end{array}$ & $22 / 22$ \\
\hline Tillack et al. [23] & Germany & Adult & Established & MRE & 19 & $18 / 19$ & 0 & Enteroclysis & $\begin{array}{l}>4 \mathrm{~mm} \text { SB wall } \\
\text { thickening \& } \\
\text { enhancement, } \\
\text { submucosal } \\
\text { edema, deep } \\
\text { ulcers/fissures, } \\
\text { cobblestone } \\
\text { pattern, enhanced } \\
\text { LNs }\end{array}$ & $18 / 19$ & - & - \\
\hline Böcker et al. [21] & Germany & Adult & Both & MRE & 21 & $9 / 21$ & NS & Enterography & $\begin{array}{l}\text { SB wall thickening } \\
\text { \& enhancement, } \\
\text { edema, mesenteric } \\
\text { injection, 'creeping } \\
\text { fat sign', prominent } \\
\text { LNs }\end{array}$ & $6 / 21$ & - & - \\
\hline Petruzziello et al. [30] & Italy & Adult & Established & US & 32 & $30 / 32$ & 1 & - & - & - & $\begin{array}{l}\geq 3 \mathrm{~mm} \mathrm{SB} \text { wall } \\
\text { thickening, } \\
\text { "stiff loop", SB } \\
\text { dilation } \\
>2.5 \mathrm{~cm} \text {, } \\
\text { stricture }<1 \mathrm{~cm} \text {, } \\
\text { fistulas, } \\
\text { abscesses }\end{array}$ & $30 / 32$ \\
\hline Casciani et al. [27] & Italy & Paediatric & Suspected & MRE & 60 & $10 / 37$ & 0 & Enterography & $\begin{array}{l}>3 \mathrm{~mm} \text { SB wall } \\
\text { thickening \& } \\
\text { enhancement, } \\
\text { oedema, stratified } \\
\text { appearance on } \\
\text { contrast-enhanced } \\
\text { T1-weighted } \\
\text { fat-suppressed, } \\
\text { stricture, comb } \\
\text { sign, enhanced LNs, } \\
\text { fistula, abscess, } \\
\text { intraperitoneal } \\
\text { fluid }\end{array}$ & $19 / 60$ & - & - \\
\hline
\end{tabular}




\begin{tabular}{|c|c|c|c|c|c|c|c|c|c|c|c|c|}
\hline Jensen et al. [18] & Denmark & Adult & Both & MRE & 93 & $24 / 80$ & 0 & Enterography & $\begin{array}{l}\text { Mucosal ulceration, } \\
\geq 6 \mathrm{~mm} \text { SB wall } \\
\text { thickening \& } \\
\text { enhancement, } \\
\text { stenosis, creeping } \\
\text { fat, dilated vasa } \\
\text { recta, abscess, fistula }\end{array}$ & $22 / 80$ & - & - \\
\hline Petruziello et al. [29] & Italy & Adult & Suspected & US & 30 & $12 / 30$ & 1 & - & - & - & $\begin{array}{l}\geq 3 \mathrm{~mm} \text { SB wall } \\
\text { thickening, "stiff } \\
\text { loop", SB } \\
\text { dilation }>2.5 \mathrm{~cm} \text {, } \\
\text { stricture }<1 \mathrm{~cm} \text {, } \\
\text { fistulas, } \\
\text { abscesses }\end{array}$ & $12 / 30$ \\
\hline Wiarda et al. [19] & $\begin{array}{l}\text { The } \\
\text { Netherlands }\end{array}$ & Adult & Both & MRE & 38 & $6 / 25$ & 1 & Enteroclysis & $\begin{array}{l}>4 \mathrm{~mm} \text { SB wall } \\
\text { thickening, } \\
\text { intramural \& } \\
\text { mesenteric edema, } \\
\text { mucosal hyperemia, } \\
\text { wall enhancement, } \\
\text { ulcerations, fistula }\end{array}$ & $16 / 38$ & - & - \\
\hline Aloi et al. [25] & Italy & Paediatric & Both & MRE \& US & 25 & $16 / 25$ & 0 & Enterography & $\begin{array}{l}>3 \mathrm{~mm} \text { SB wall } \\
\text { thickening \& } \\
\text { enhancement, } \\
\text { edema, stratified } \\
\text { appearance on } \\
\text { contrast-enhanced } \\
\text { T1-weighted } \\
\text { fat-suppressed, } \\
\text { strictures }<10 \mathrm{~mm} \text {, } \\
\text { comb sign, enhanced } \\
\text { LNs, fistula, abscess, } \\
\text { intraperitoneal fluid }\end{array}$ & $15 / 25$ & $\begin{array}{l}>3 \mathrm{~mm} \text { SB wall } \\
\text { thickening, loss } \\
\text { of stratification } \\
\text { of bowel wall, } \\
\text { "stiffness", } \\
\text { strictures, thick- } \\
\text { ened/hyperechoic } \\
\text { mesentery, } \\
\text { enlarged LNs, } \\
\text { stenosis }<1 \mathrm{~cm} \text {, } \\
\mathrm{SB} \text { dilatation } \\
>2.5 \mathrm{~cm}\end{array}$ & $16 / 25$ \\
\hline Kopylov et al. [31] & Israel & Adult & Established & MRE & 77 & $44 / 52$ & $\begin{array}{l}0(17 \mathrm{pts} \\
\text { excluded } \\
\text { following } \\
\text { patency capsule })\end{array}$ & Enterography & $\begin{array}{l}>3 \mathrm{~mm} \text { SB wall } \\
\text { thickening \& } \\
\text { enhancement, } \\
\text { luminal stenosis } \\
>80 \% \text {, pre-stenotic } \\
\text { dilatation }>2.5 \mathrm{~cm}\end{array}$ & $40 / 52$ & - & - \\
\hline Oliva et al. [28] & Italy & Paediatric & Established & MRE \& US & 38 & $\begin{array}{l}19 / 38 \\
{ }^{*} \mathrm{CCE}\end{array}$ & 0 & Enterography & $\begin{array}{l}>3 \mathrm{~mm} \text { SB wall } \\
\text { thickening \& } \\
\text { enhancement, } \\
\text { edema, stratified } \\
\text { appearance on } \\
\text { contrast-enhanced } \\
\text { T1-weighted } \\
\text { fat-suppressed, } \\
\text { strictures }<10 \mathrm{~mm} \text {, } \\
\text { comb sign, enhanced } \\
\text { LNs, fistula, abscess, } \\
\text { intraperitoneal fluid }\end{array}$ & $19 / 38$ & NS (standard) & $21 / 38$ \\
\hline
\end{tabular}


a

$\begin{array}{cccc} & \text { CE } & \text { MRE } & \text { Odds Ratio } \\ \text { Study or Subgroup } & \text { Events Total Events Total Weight } & \text { M-H, Random, } 95 * \mathrm{Cl}\end{array}$

\begin{tabular}{|c|c|c|c|c|c|}
\hline Albert 2005 & 25 & 27 & 35 & 52 & 5 \\
\hline Aloi 2015 & 16 & 25 & 15 & 25 & \\
\hline Bocker 2010 & 9 & 2 & 6 & 21 & \\
\hline Casciani 2011 & 10 & 37 & 19 & 60 & \\
\hline Gölder zoos & 11 & 15 & 8 & 15 & \\
\hline Jensen 2011 & 24 & 80 & 22 & 80 & \\
\hline Kopylov 2015 & 44 & 52 & 40 & 52 & \\
\hline Oliva 2016 & 18 & 38 & 19 & 38 & \\
\hline Tillack 2008 & 18 & 19 & 18 & 19 & \\
\hline Wiarda 2011 & 6 & 25 & 16 & 38 & \\
\hline Total $(95 \% \mathrm{Cl})$ & & 339 & & $\$ 00$ & \\
\hline Total events & 182 & & 199 & & \\
\hline
\end{tabular}

$6.07[1.28,28.68]$

$1.19[0.38,3.72]$

$1.88[0.52,6.76]$

$0.80[0.32,1.98]$

$1.83[0.39,8.57]$

$1.13[0.57,2.24]$

$1.65[0.81,4.45]$

$1.00[0.41,2.46]$

$1.00[0.06,17.25]$

$0.43[0.14,1.33]$

$1.17[0.83,1.67]$
Odds Ratio

M. $-\mathrm{H}$, Random, $95 \% \mathrm{Cl}$

b

CE

US

Odds Ratio

Study or Subgroup Events Total Events Total Weight $\mathrm{M}-\mathrm{H}$, Fixed, $95 \% \mathrm{Cl}$

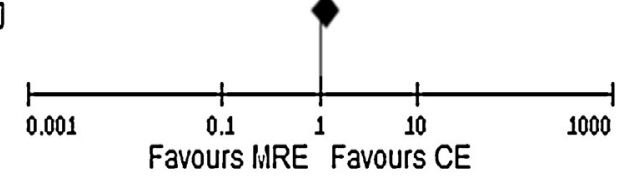

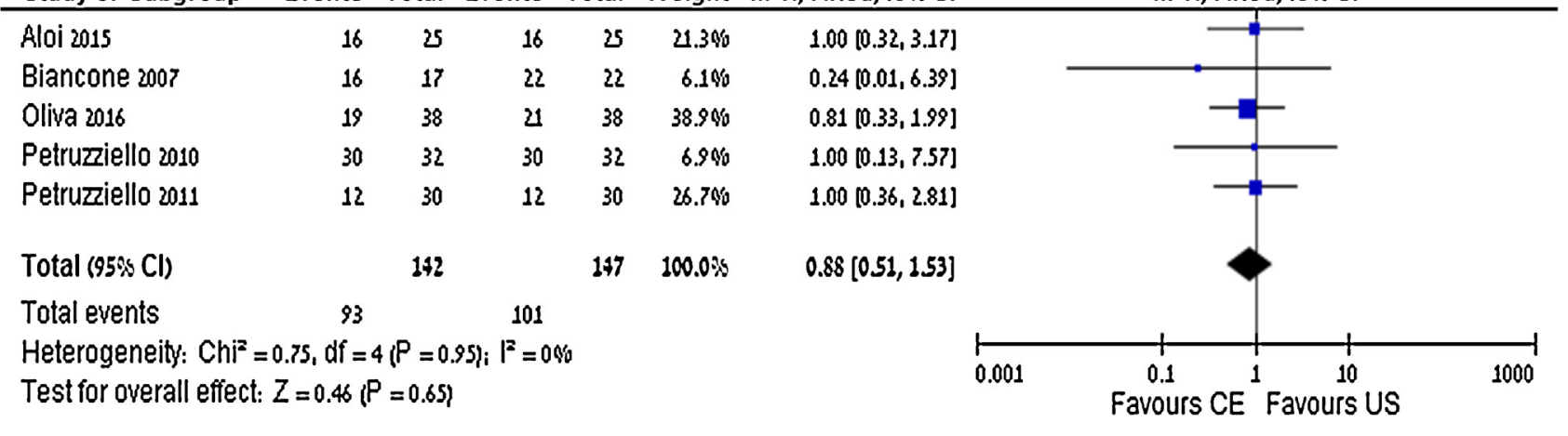

Fig. 2. Diagnostic yield of capsule endoscopy, magnetic resonance enterography and ultrasound for small bowel Crohn's disease. (a) CE vs MRE; (b) CE vs SICUS.

established CD only [20,22,23,28,30]; the rest included both suspected and established $\mathrm{CD}$. Two studies compared all three of the modalities [25,28], while 8 compared CE to MRE and 3 to SICUS.

\subsection{Comparison of diagnostic modalities}

3.2.1. Primary analysis: CE vs MRE and US for detection of small bowel disease in both suspected and established $C D$

On per protocol analysis, the DY of CE was similar to that of MRE (10 studies, 400 patients, OR 1.17 ; 95\% CI 0.83-1.67; $P=0.37 ; I^{2}=5 \%$ ) and SICUS (5 studies, 142 patients, OR 0.88; 95\% CI $0.51-1.53 ; P=0.65 ; I^{2}=0 \%$ ) (Fig. 2). The QUADAS-2 analysis is shown in Table 2; studies were generally of good quality with low risk of bias.

\subsubsection{Secondary analyses}

The DY of CE was similar to that of MRE ( 2 studies, 85 patients, OR 3.24; 95\% CI 0.14-72.76; $P=0.46 ; I^{2}=86 \%$ ) and SICUS ( 1 study, 30 patients, OR $1.00 ; 95 \%$ CI $\left.0.36-2.81 ; P=1.00 ; I^{2}=N A\right)$ for suspected $\mathrm{CD}$; results were similar for established CD compared to MRE ( 5 studies, 152 patients, OR $0.88 ; 95 \%$ CI $\left.0.53-1.48 ; P=0.63 ; I^{2}=48 \%\right)$ and SICUS ( 3 studies, 92 patients, OR 0.57; 95\% CI 0.27-1.20; $P=0.09 ; I^{2}=67 \%$ ) (Fig. 3). Furthermore, the DY of CE was also similar to MRE when the analysis was stratified by age group (adult vs pediatric) (Fig. 4) or when limited to studies with low risk of bias (Supplemental material).
CE was superior to MRE for the detection of proximal SB disease (7 studies, 251 patients, OR 2.79; 95\% CI 1.2-6.48; $P=0.02$; $I^{2}=68 \%$;); this was not significantly different to distal SB DY (7 studies, 251 patients, OR 0.91; 95\% CI 0.50-1.63; $P=0.09 ; I^{2}=67 \%$;). There was a trend for a superior accuracy for CE vs US for detection of proximal small bowel disease, however the comparison did not reach statistical significance and was based on a small number of patients ( 3 studies, 95 patients, OR 2.76; 95\% CI 0.84-9.02; $P=0.09$; $I^{2}=67 \%$; (Fig. 5).

The results remained similar when the analyses were repeated for ITT instead of per-protocol analysis (Supplemental material).

\section{Discussion}

As the SB is involved in over 70\% of CD patients, its thorough evaluation is vital for initial diagnosis, establishing disease phenotype, and assessment of mucosal healing and prognosis [31-33]. Previous work has shown that CE was superior to CTE and SBFT, but not MRE [3]. Since then, several further studies have been published.

The results of our meta-analysis suggest a similar diagnostic yield for detection of SB inflammation by CE, MRE and SICUS. Therefore no diagnostic modality can currently be considered a "gold-standard" for SB evaluation. The accuracy was similar for patients with suspected and established CD. However, the superior accuracy of CE for detection of proximal SB disease may have an important prognostic value, as proximal SB involvement is asso- 
a

CE

MRE

Odds Ratio

Odds Ratio

Study or Subgroup Events Total Events Total Weight $\mathrm{M} \cdot \mathrm{H}$, Random, $95 \% \mathrm{Cl}$

\begin{tabular}{|c|c|c|c|c|c|c|c|c|}
\hline Albert 2005 & 12 & 13 & 10 & 25 & $44.9 \%$ & $18.00[2.01,161.04]$ & & $\longrightarrow$ \\
\hline Casciani 2011 & 10 & 37 & 19 & 60 & $55.1 \%$ & $0.80[0.32,1.98]$ & & \\
\hline Total (95\% Cl) & & 50 & & 85 & $100.0 \%$ & $3.24[0.14,72.76]$ & & \\
\hline Total events & 22 & & $\vartheta$ & & & & & \\
\hline $\begin{array}{l}\text { Heterogeneity: Tau² } \\
\text { Test for overall effect: }\end{array}$ & $\begin{array}{l}=4.36_{\mathrm{i}} \mathrm{Chi}^{2} \\
\mathrm{Z}=0.74 \mathrm{P}\end{array}$ & $\begin{array}{l}=6.96 . \\
=0.461\end{array}$ & $P=$ & 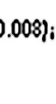 & - & & Favours MRE & $1 \frac{10}{\text { Favours CE }}$ \\
\hline
\end{tabular}

CE

US

Odds Ratio

Odds Ratio

Study or Subgroup Events Total Events Total Weight $\mathrm{M} \cdot \mathrm{H}$, Random, $95 \% \mathrm{Cl}$ $\mathrm{M} \cdot \mathrm{H}$, Random, $95 \% \mathrm{Cl}$

$\begin{array}{lccccc}\text { Petruzziello } 2011 & 12 & 30 & 12 & 30 & 100.0 \% \\ & & 30 & & 30 & 100.0 \% \\ \text { Total }(95 \% \mathrm{Cl}) & 12 & & 12 & & \\ \text { Total events } & 12 & & & & \\ \begin{array}{l}\text { Heterogeneity: Not applicable } \\ \text { Test for Overall effect: } Z=0.00(P=1.00)\end{array} & & & \end{array}$

$1.00[0.36,2.81$

$1.00[0.36,2.81]$

Test for overall effect: $Z=0.00(P=1.00)$

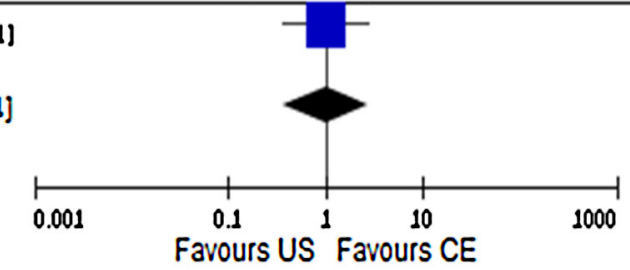

b

Experimental Control Odds Ratio

Study or Subgroup Events Total Events Total Weight $\mathrm{M} \cdot \mathrm{H}$, Fixed, $95 \% \mathrm{Cl}$

Odds Ratio

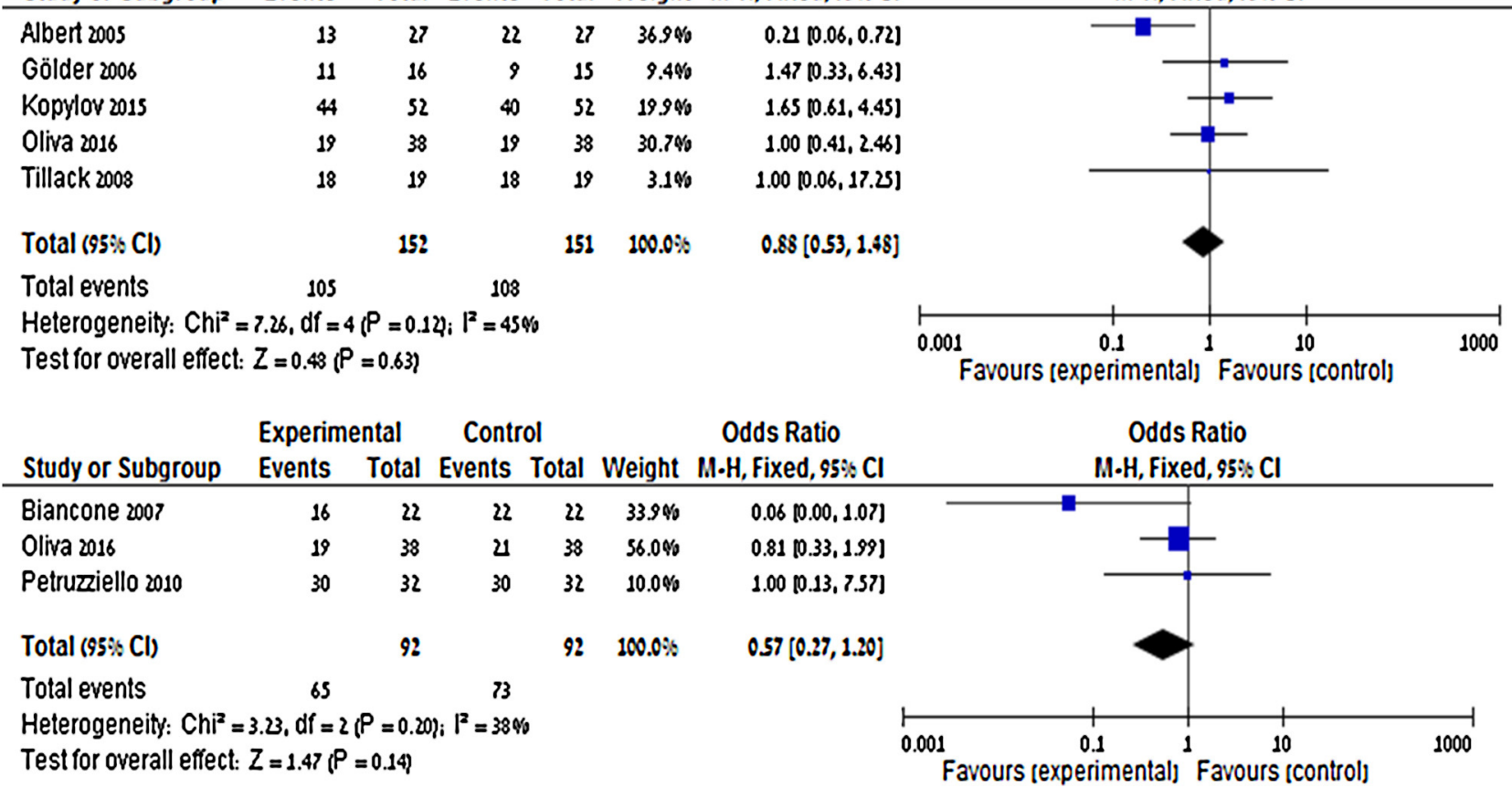

Fig. 3. Diagnostic yield of capsule endoscopy, magnetic resonance enterography and ultrasound for suspected (a) and established (b) small bowel Crohn's disease.

Table 2

Quality assessment of diagnostic accuracy studies (QUADAS) 2 for the included studies. $\ominus$ denotes significant risk of bias, ? unclear risk of bias and $\odot$ low risk of bias.

\begin{tabular}{|c|c|c|c|c|c|c|c|}
\hline Author, reference & $\begin{array}{l}\text { Item 1: risk of bias } \\
\text { in pt selection? }\end{array}$ & $\begin{array}{l}\text { Item } 2 \text { : } \\
\text { representative } \\
\text { pt spectrum? }\end{array}$ & $\begin{array}{l}\text { Item 3: risk of bias } \\
\text { in conduct or } \\
\text { interpretation of } \\
\text { index test (MRE } \\
\text { and/or SICUS)? }\end{array}$ & $\begin{array}{l}\text { Item 4: } \\
\text { applicability of } \\
\text { index test (MRE } \\
\text { and/or SICUS) to } \\
\text { review question? }\end{array}$ & $\begin{array}{l}\text { Item } 5 \text { : risk of bias } \\
\text { from conduct or } \\
\text { interpretation of } \\
\text { reference standard } \\
(\mathrm{CE}) \text { ? }\end{array}$ & $\begin{array}{l}\text { Item } 6 \text { : does the } \\
\text { target condition } \\
\text { match the review } \\
\text { question? }\end{array}$ & $\begin{array}{l}\text { Item 7: risk of } \\
\text { bias from pt } \\
\text { flow? }\end{array}$ \\
\hline 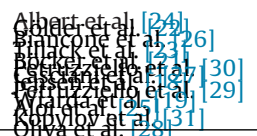 & 育 & 䒺 & 촛 & 촟 & 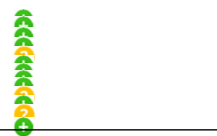 & 촟 & 촟 \\
\hline
\end{tabular}

Abbreviations: CE, capsule endoscopy; MRE, magnetic resonance enterography; pt, patient; SICUS, small intestinal contrast ultrasonography. 
a

CE MRE

Odds Ratio

Odds Ratio

Study or Subgroup Events Total Events Total Weight $\mathrm{M} \cdot \mathrm{H}$, Random, $95 \% \mathrm{Cl}$

$\mathrm{M} \cdot \mathrm{H}$, Random, $95 \% \mathrm{Cl}$

\begin{tabular}{|c|c|c|c|c|c|}
\hline \multicolumn{6}{|l|}{ 1.11.1 Adults } \\
\hline Albert 2005 & 25 & 27 & 35 & 52 & $5.0 \%$ \\
\hline Bocker 2010 & 9 & 2 & 6 & 21 & $7.2 \%$ \\
\hline Gölder 2006 & 11 & 15 & 8 & 15 & $5.1 \%$ \\
\hline Jensen 2011 & 24 & 80 & 22 & 80 & $23.1 \%$ \\
\hline Kopylov 2015 & 44 & 52 & 40 & 52 & $11.8 \%$ \\
\hline Tillack 2008 & 18 & 19 & 18 & 19 & $1.5 \%$ \\
\hline $\begin{array}{l}\text { Wiarda } 2011 \\
\text { Subtotal }(95 \% \mathrm{Cl})\end{array}$ & 6 & $\begin{array}{r}25 \\
239\end{array}$ & 16 & $\begin{array}{r}38 \\
277\end{array}$ & $\begin{array}{l}9.3 \% \\
63.0 \%\end{array}$ \\
\hline \multicolumn{6}{|c|}{$\begin{array}{l}\text { Heterogeneity: } \operatorname{Tau}^{2}=0.15 ; \mathrm{Chi}^{2}=8.41, \mathrm{df}=\left.\sigma(P=0.21)_{i}\right|^{2}=\mho \vartheta \% \\
\text { Test for overall effect: } Z=1.15(P=0.25)\end{array}$} \\
\hline \multicolumn{6}{|l|}{ 1.11.2 Pediatrics } \\
\hline Aloi 2015 & 16 & 25 & 15 & 25 & $9.0 \%$ \\
\hline Casciani 2011 & 10 & 37 & 19 & 60 & $13.9 \%$ \\
\hline $\begin{array}{l}\text { Oliva } 2016 \\
\text { Subtotal }(95 \% \mathrm{Cl})\end{array}$ & 19 & $\begin{array}{r}38 \\
100\end{array}$ & 19 & $\begin{array}{r}38 \\
123\end{array}$ & $\begin{array}{l}14.1 \% \\
37.0 \%\end{array}$ \\
\hline \multicolumn{6}{|c|}{$\begin{array}{l}\text { Heterogeneity: } \operatorname{Tau}^{2}=0.00 ; \mathrm{Chi}^{2}=0.30, \mathrm{df}=\left.2(\mathrm{P}=0.86)_{;}\right|^{2}=0 \% \\
\text { Test for overall effect: } Z=0.16(P=0.88)\end{array}$} \\
\hline Total $(95 \% \mathrm{Cl})$ & \multicolumn{3}{|c|}{339} & 400 & $100.0 \%$ \\
\hline Total events & 182 & & 198 & & \\
\hline \multicolumn{6}{|c|}{$\begin{array}{l}\text { Heterogeneity: } \text { Tau }^{2}=0.0 z_{i} C h i^{2}=9.48, d f=\left.9(P=0.39)_{i}\right|^{2}=5 \% \\
\text { Test for overall effect: } Z=0.89(P=0.37 \\
\text { Test for subaroun differences: }\left.C h\right|^{2}=0.83, d f=1(P=0.36),\left.\right|^{2}=0 \%\end{array}$} \\
\hline
\end{tabular}

$6.07[1.28,28.68]$

$1.88[0.52,6.76]$

$1.83[0.39,8.57]$

$1.13[0.57,2.24]$

$1.65[0.61,4.45]$

$1.00[0.06,17.25]$

$0.43[0.14,1.33]$

$1.37[0.80,2.36]$
$1.19[0.38,3.72]$

$0.80[0.32,1.98]$

$1.00[0.41,2.48]$

$0.96[0.55,1.67]$

$1.17[0.83,1.67]$

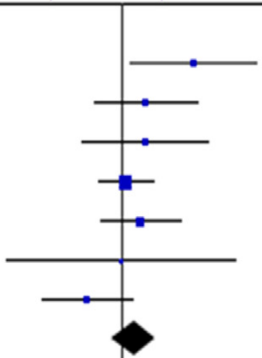

b

CE

US

Odds Ratio

Study or Subgroup Events Total Events Total Weight $\mathrm{M} \cdot \mathrm{H}$, Fixed, $95 \% \mathrm{Cl}$ 2.9.1 Adults

Biancone 2007

22

$22 \quad 6.1 \%$

$0.24[0.01,6.39]$

Petruzziello 2010

3032

3032

$6.9 \%$

$1.00[0.13,7.57]$

Petruzziello 2011

$\begin{array}{lllll}12 & 30 & 12 & 30 & 26.7 \%\end{array}$

$1.00[0.36,2.81]$

Subtotal $(95 \% \mathrm{Cl})$

79

$84 \quad 39.7 \%$

$0.88[0.37,2.12]$

Total events

$58 \quad 64$

Heterogeneity: $\mathrm{Chi}^{2}=0.67 \mathrm{df}=2\left(\mathrm{P}=0.7 \eta_{i} \mathrm{l}^{2}=0 \%\right.$

Test for overall effect: $Z=0.28(P=0.78)$

\subsubsection{Pediatrics}

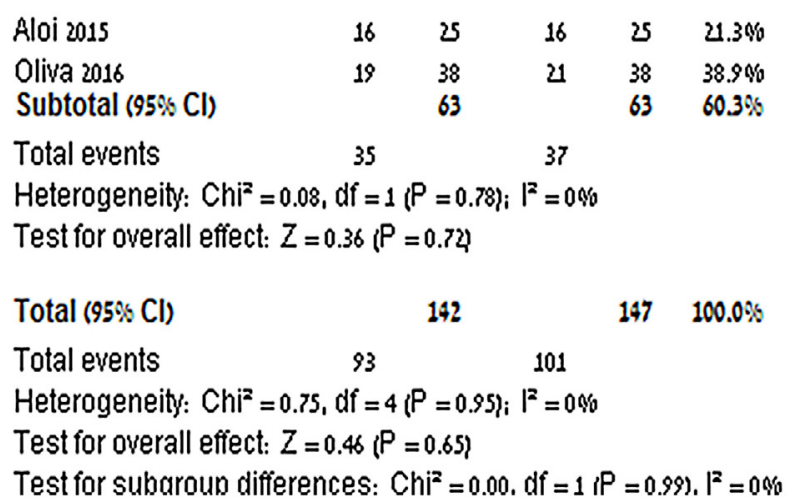

$1.00[0.32,3.17]$

$0.81[0.33,1.99]$

$0.88[0.43,1.78]$

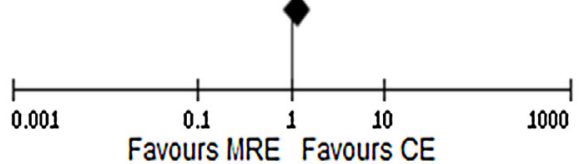

Favours MRE Favours CE

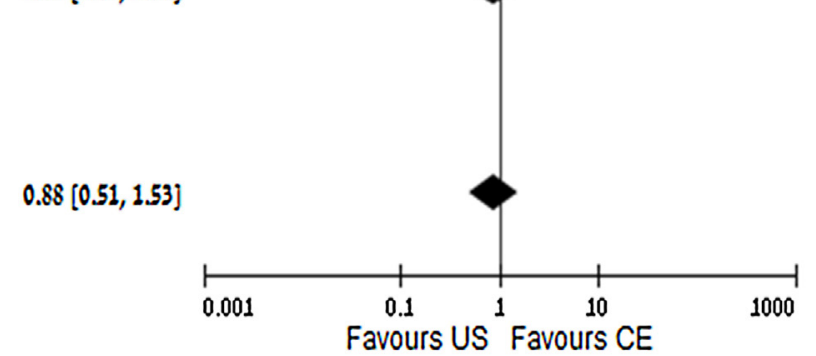

Fig. 4. Diagnostic yield of capsule endoscopy, magnetic resonance enterography and ultrasound for small bowel Crohn's disease in pediatric and adult patients (a) CE vs MRE; (b) CE vs SICUS. 
a

Study or Subgroup Events Total Events Total Weight $\mathrm{M} \cdot \mathrm{H}$, Random, $95 \% \mathrm{Cl}$

\begin{tabular}{|c|c|c|c|c|c|}
\hline Aloi 2015 & 18 & 25 & 17 & 25 & $15.2 \%$ \\
\hline Bocker 2010 & 7 & 2 & 5 & $u$ & $14.1 \%$ \\
\hline Gölder 2006 & 8 & 16 & 1 & 16 & $8.6 \%$ \\
\hline Jensen 2011 & 18 & 80 & 2 & 80 & $13.0 \%$ \\
\hline Kopylov 2015 & 32 & 52 & 12 & 52 & $18.0 \%$ \\
\hline Oliva 2016 & 14 & 38 & 13 & 28 & $16.9 \%$ \\
\hline Tillack 2008 & 13 & 19 & 8 & 18 & 14.340 \\
\hline Total (95\% Cl) & & 251 & & 241 & $100.0 \%$ \\
\hline Total events & 111 & & 59 & & \\
\hline
\end{tabular}

CE

US

Study or Subgroup Events Total Events Total Weight $\mathrm{M} \cdot \mathrm{H}$, Random, $95 \% \mathrm{Cl}$

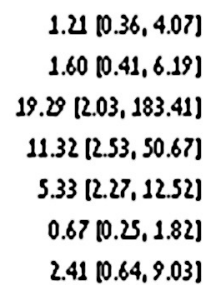

$2.79[1.20,6.48]$

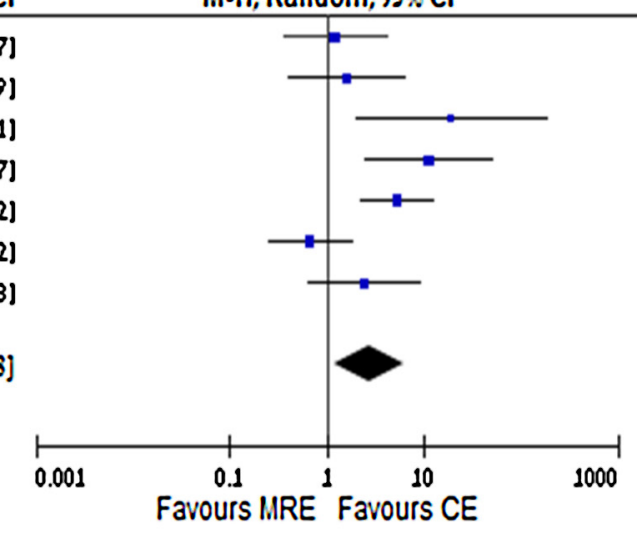

Odds Ratio

Odds Ratio

$\mathrm{M} \cdot \mathrm{H}$, Random, $95 \% \mathrm{Cl}$

\begin{tabular}{|c|c|c|c|c|c|}
\hline Aloi 2015 & 18 & 2 & 13 & 25 & $33.5 \%$ \\
\hline Oliva 2016 & 11 & 38 & 10 & 38 & $36.7 \%$ \\
\hline Petruzziello 2010 & 16 & 32 & 3 & 32 & $8.8 \%$ \\
\hline Total (95\% Cl) & & 95 & & 95 & $100.0:$ \\
\hline Total events & 45 & & 28 & & \\
\hline Heterogeneity: $T$ & Chi & 8.07. & $2(P=$ & $.05\}$ & $=67 \%$ \\
\hline
\end{tabular}

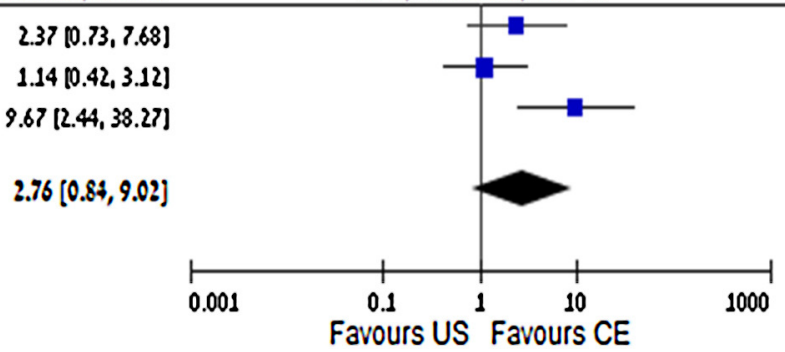

b

CE MRE

Odds Ratio

Study or Subgroup Events Total Events Total Weight $\mathrm{M} \cdot \mathrm{H}$, Random, $95 \% \mathrm{Cl}$

Odds Ratio

\begin{tabular}{|c|c|c|c|c|c|c|c|c|c|}
\hline Aloi 2015 & 14 & 25 & 17 & 25 & $15.1 \%$ & $0.60[0.19,1.90]$ & & $\rightarrow-$ & \\
\hline Bocker 2010 & 1 & u & 6 & 2 & $5.8 \%$ & $0.13[0.01,1.15]$ & & & \\
\hline Gölder 2006 & 9 & 16 & 9 & 16 & $11.8 \%$ & $1.00[0.25,4.04]$ & & & \\
\hline Jensen 2011 & 24 & 80 & 20 & 80 & $24.0 \%$ & $1.20[0.64,2.58]$ & & & \\
\hline Kopylov 2015 & 35 & 52 & 39 & 52 & $20.5 \%$ & $0.69[0.28,1.61]$ & & & \\
\hline Oliva 2016 & 11 & 18 & 12 & 38 & $14.8 \%$ & $3.40[1.06,10.86]$ & & & \\
\hline Tillack 2003 & 15 & 18 & 17 & 19 & $8.0 \%$ & $0.44[0.07,2.76]$ & & & \\
\hline Total $(95 \% \mathrm{Cl})$ & & 231 & & 251 & $100.0 \%$ & $0.91[0.50,1.63]$ & & & \\
\hline \multirow{2}{*}{\multicolumn{7}{|c|}{$\begin{array}{l}\text { Total events } \quad 109 \quad 120 \\
\text { Heterogeneity: Tau }=0.24 ; C h i^{2}=10.37, d f=6(P=0.1)_{i} I^{2}=42 \% \\
\text { Test for overall effect: } Z=0.33(P=0.74)\end{array}$}} & & & \\
\hline & & & & & & & 0.001 & $\begin{array}{c}0.1 \\
\text { Favours MRE }\end{array} \stackrel{10}{10}$ Favours CE & 1000 \\
\hline & CE & & US & & & Odds Ratio & & Odds Ratio & \\
\hline Study or Subgroup & Events & Total & Events & Total & Weight & $\mathrm{M} \cdot \mathrm{H}$, Fixed, $95 \% \mathrm{Cl}$ & & $\mathrm{M} \cdot \mathrm{H}$, Fixed, $95 \% \mathrm{Cl}$ & \\
\hline Aloi 2015 & 14 & 25 & 17 & 25 & $68.3 \%$ & $0.60[0.19,1.90]$ & & & \\
\hline Oliva 2016 & 15 & 18 & 18 & 38 & $17.1 \%$ & $5.56[1.38 .22 .38]$ & & & \\
\hline Petruziello 2010 & 30 & 32 & 30 & 32 & $16.6 \%$ & $1.00[0.13,7.57]$ & & & \\
\hline Total (95\% Cl) & & 75 & & 95 & $100.0 \%$ & $1.51[0.72,3.20]$ & & & \\
\hline Total events & 58 & & 65 & & & & & & \\
\hline $\begin{array}{l}\text { Heterogeneity: } \mathrm{Chi}^{2} \\
\text { Test for overall effect }\end{array}$ & $\begin{array}{l}5.99, d f= \\
Z=1.08 \text { if }\end{array}$ & $\begin{array}{l}2(P=0.4 \\
=0.28)\end{array}$ & $.05) ; F^{2}=67$ & & & & 0.001 & $\begin{array}{l}0.1 \\
\text { Favours US }\end{array} \stackrel{1}{10}$ Favours CE & 1000 \\
\hline
\end{tabular}

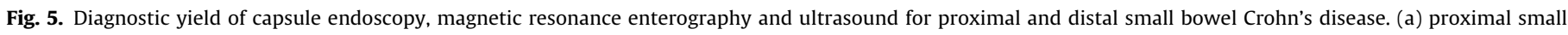
bowel (b) distal small bowel. 
ciated with a higher risk of surgery [34]. This disease location is frequently underestimated during the initial assessment; nevertheless, CE may detect active inflammation in the jejunum or proximal ileum in over $50 \%$ of patients with established CD [35]. The currently accepted Montreal classification does not specifically address proximal SB disease location [36], which is better reflected in the pediatric Paris classification (L4b) [37]. The assessment of disease severity and extent is essential for diagnostic categorization and management planning in pediatric inflammatory bowel disease. Indeed, in the latest guidelines for management and diagnosis of pediatric IBD, SB evaluation is encouraged in all of the patients with suspected IBD, and it is essential in pediatric patients with established CD, IBD-U, or atypical UC. For this reason, in children the higher DY and the lack of invasiveness of CE should be taken into account before SB evaluation. Conversely, there is no evidence to support modification of the treatment strategy in adult patients with proximal SB disease.

Diagnostic modalities evaluated in our meta-analysis are all well-established and extensively evaluated for detection and follow-up of small bowel Crohn's disease .The current ECCO/ESGAR guidelines support the use of MRE, US and CE for these purposes, although evaluation of small bowel patency is required before utilization of $\mathrm{CE}$ in established $\mathrm{CD}$ [35]. The main purposes of diagnostic evaluation in established $C D$ are validation of disease characteristics, establishement of prognosis, monitoring of mucosal healing and identification of complications [7,20,31,32,38-41]. All the evaluated diagnostic modalities have their distinct diagnostic strengths and disadvantages. However, selection of a diagnostic modality takes into consideration additional variables such as local expertise, availability and resources. A recent Italian study comparing diagnostic strategies for CD suggested that ileocolonoscopy followed by SICUS may be the most cost-effective strategy [15]; however this may not necessarily hold true when different reimbursement schemes or different pretest probabilities are considered. Moreover, patient preference may have a major impact on adherence with any monitoring strategy, especially since $C D$ patients are likely to require multiple diagnostic procedures during the course of the disease. A recent study that compared patients' discomfort associated with MRE and CE clearly demonstrated that CE was significantly better accepted and associated with less discomfort both during preparation; moreover, the patents were more likely to agree to $C E$ as a follow-up procedure [9]. As CE is associated with a certain risk of capsule retention in patients with SB CD, an evaluation of SB patency with crosssectional imaging is recommended before the procedure [42]. This approach lead to a significant decrease in a rate of capsule retentions reported in the recent literature (1.3-2.6\%) as compared to the older studies that suggested a much higher risk of retention of up to $13 \%[31,36,43-47]$. The perceived risk of retention lead to the exclusion of patients with suspected strictures from most of the studies; although in some of the studies imaging results were available for all patients, the majority reported both $\mathrm{CE}$ and imaging results only for patients eligible for all modalities. Therefore, in this metaanalysis we have used both a "per-protocol" and "ITT" analysis where possible, with no significant changes between the analytical strategies. Importantly, the patency capsule was used in only 2 studies [19,20]; capsule retentions were rare (pooled incidence of $4 / 500$ patients, $0.8 \%$ ) and consistent with previous studies [31].

Most of the limitations of our study are inherent to all diagnostic meta-analyses and include heterogeneity in diagnostic protocols, diagnostic criteria and patient selection. There was lack of a "goldstandard" modality for detection of SB CD, therefore most of the included studies compared the modalities against each other. Thus, a calculation of estimated sensitivity and specificity for the modalities was impossible due to a lack of gold-standard modality for which the results obtained by either modality could be compared.
An additional limitation of our analysis is that we limited it to studies using CE as a comparator. Alternative diagnostic techniques for the modalities discussed in the study are available and some are well established for diagnosis and monitoring of $\mathrm{CD}$ (such as diffusion weighted MRE, bubble-enhanced or non-contrast enhanced intestinal US), however we could not discuss those as there was no sufficient data for comparison with CE [48-50]. As for CT enterography, a previous analysis by Dionisio et al. performed a thorough analysis of its accuracy as compared to VCE [3]. With the diminishing use of CTE for routine CD evaluation due to a concern of radiation exposure and a small number of new comparative studies, we considered an additional analysis to be redundant at this point.

Several studies chose to compare the results to an "expert panel" who corroborated the results of all diagnostic tests with clinical follow-up; the validity of this strategy is unclear; however it should be noted that $C D$ is routinely diagnosed using a constellation of clinical, endoscopic and imaging criteria (e.g. the Lennard-Jones criteria) [51] and not by a single finding. This is reflected in the heterogeneity of clinical disease definitions of different modalities, especially for CE. Most studies did not use a validated endoscopic score (Lewis score [52] or Crohn's disease capsule disease activity index [53]) but relied on pathognomonic features which may be non-specific in milder cases. This limitation also limited our ability to discuss the diagnostic accuracy for evaluation of mucosal healing Moreover, the MRE protocols and sequences differed between the studies. Conversely, all studies comparing CE to US originated from a single country (Italy) and implied a single technique (SICUS). Thus, these results are not applicable to other intestinal ultrasound techniques. In summary, our results demonstrate that CE, MRE and SICUS have similar DY for the detection of SB CD; none of the modalities can be considered superior over the others and their utilization should be tailored to the specific clinical situations. CE is the preferred modality for detection of proximal small bowel involvement. This advantage needs to be weighed against a small, but non-negligible risk of capsule retention

\section{Conflicts of interest}

None declared.

\section{Appendix A. Supplementary data}

Supplementary data associated with this article can be found, in the online version, at http://dx.doi.org/10.1016/j.dld.2017.04.013.

\section{References}

[1] Cosnes J, Gowerrousseau C, Seksik P, et al. Epidemiology and natural history of inflammatory bowel diseases. Gastroenterology 2011;140:1785-94

[2] Panes J, Bouhnik Y, Reinisch W, et al. Imaging techniques for assessment of inflammatory bowel disease: joint ECCO and ESGAR evidence-based consensus guidelines. Journal of Crohn's and Colitis 2013:7:556-85.

[3] Dionisio PM, Gurudu SR, Leighton JA, et al. Capsule endoscopy has a significantly higher diagnostic yield in patients with suspected and established small-bowel Crohn's disease: a meta-analysis. The American Journal of Gastroenterology 2010;105:1240-8

[4] Higgins JPT, Thompson SG. Quantifying heterogeneity in a meta-analysis. Statistics in Medicined 2002;21:1539-58.

[5] Misangyi VF, LePine JA, Algina J, et al. The adequacy of repeated-measures regression for multilevel research: comparisons with repeated-measures ANOVA, multivariate repeated-measures ANOVA, and multilevel modeling across various multilevel research designs. Organizational Research Methods 2006;9:5-28.

[6] Whiting PF, Rutjes AWS, Westwood ME, et al. Quadas-2: a revised tool for the quality assessment of diagnostic accuracy studies. Annals of Internal Medicine 2011:155:529-36.

[7] Greener T, Klang E, Yablecovitch D, et al. The impact of magnetic resonance enterography and capsule endoscopy on the re-classification of disease in patients with known Crohn's disease: a prospective Israeli IBD research nucleus (IIRN) study. Journal of Crohn's and Colitis 2016;10:525-31.

[8] Kopylov U, Klang E, Yablecovitch D, et al. Magnetic resonance enterography versus capsule endoscopy activity indices for quantification of small bowel 
inflammation in Crohn's disease. Therapeutic Advances in Gastroenterology 2016;9:655-63.

[9] Lahat A, Kopylov U, Amitai MM, et al. Magnetic resonance enterography or video capsule endoscopy-what do Crohn's disease patients prefer. Patient Preference and Adherence 2016;10:1043-50.

[10] Rozendorn N, Klang E, Lahat A, et al. Prediction of patency capsule retention in known Crohn's disease patients by using magnetic resonance imaging. Gastrointestinal Endoscopy 2016;83:182-7.

[11] Van Weyenberg SJB, Bouman K, Jacobs MAJM, et al. Comparison of MR enteroclysis with video capsule endoscopy in the investigation of small-intestinal disease Abdominal Imaging 2013;38:42-51.

[12] Crook DW, Knuesel PR, Froehlich JM, et al. Comparison of magnetic resonance enterography and video capsule endoscopy in evaluating small bowel disease. European Journal of Gastroenterology and Hepatology 2009;21:54-65.

[13] Di Nardo G, Oliva S, Ferrari F, et al. Usefulness of wireless capsule endoscopy in paediatric inflammatory bowel disease. Digestive and Liver Disease 2011;43:220-4.

[14] Maccioni F, Ansari NA, Mazzamurro F, et al. Detection of Crohn disease lesions of the small and large bowel in pediatric patients: diagnostic value of MR enterography versus reference examinations. American Journal of Roentgenology 2014;203:W533-542.

[15] Maconi G, Bolzoni E, Giussani A, et al. Accuracy and cost of diagnostic strategies for patients with suspected Crohn's disease. Journal of Crohn's and Colitis 2014;8:1684-92.

[16] Rasmussen B, Nathan T, Jensen MD. Symptomatic patency capsule retention in suspected Crohn's disease. Journal of Crohn's and Colitis 2016;10:1445-7.

[17] Shiotani A, Hata J, Manabe N, et al. Clinical relevance of patency capsule combined with abdominal ultrasonography to detect small bowel strictures. European Journal of Gastroenterology and Hepatology 2014;26:1434-8.

[18] Jensen MD, Nathan T, Rafaelsen SR, et al. Diagnostic accuracy of capsule endoscopy for small bowel Crohn's disease is superior to that of MR enterography or CT enterography. Clinical Gastroenterology and Hepatology 2011;9:124-9.

[19] Wiarda BM, Mensink PBF, Heine DGN, et al. Small bowel Crohn's disease: MR enteroclysis and capsule endoscopy compared to balloon-assisted enteroscopy. Abdominal Imaging 2012;37:397-403.

[20] Kopylov U, Yablecovitch D, Lahat A, et al. Detection of small bowel mucosal healing and deep remission in patients with known small bowel Crohn's disease using biomarkers, capsule endoscopy, and imaging. American Journal of Gastroenterology 2015;110:1316-23.

[21] Böcker U, Dinter D, Litterer C, et al. Comparison of magnetic resonance imaging and video capsule enteroscopy in diagnosing small-bowel pathology: localization-dependent diagnostic yield. Scandinavian Journal of Gastroenterology 2010;45:490-500.

[22] Gölder SK, Schreyer AG, Endlicher E, et al. Comparison of capsule endoscopy and magnetic resonance (MR) enteroclysis in suspected small bowel disease. International Journal of Colorectal Disease 2006;21:97-104.

[23] Tillack C, Seiderer J, Brand S, et al. Correlation of magnetic resonance enteroclysis (MRE) and wireless capsule endoscopy (CE) in the diagnosis of small bowel lesions in Crohn's disease. Inflammatory Bowel Diseases 2008;14:1219-28.

[24] Albert JG, Martiny F, Krummenerl A, et al. Diagnosis of small bowel Crohn's disease: a prospective comparison of capsule endoscopy with magnetic resonance imaging and fluoroscopic enteroclysis. Gut 2005;54:1721-7.

[25] Aloi M, Di Nardo G, Romano G, et al. Magnetic resonance enterography, smallintestine contrast US, and capsule endoscopy to evaluate the small bowel in pediatric Crohn's disease: a prospective, blinded, comparison study. Gastrointestinal Endoscopy 2015;81:420-7.

[26] Biancone L, Calabrese E, Petruzziello C, et al. Wireless capsule endoscopy and small intestine contrast ultrasonography in recurrence of Crohn's disease. Inflammatory Bowel Diseases 2007;13:1256-65.

[27] Casciani E, Masselli G, Di Nardo G, et al. MR enterography versus capsule endoscopy in paediatric patients with suspected Crohn's disease. European Radiology 2011;2:823-31.

[28] Oliva S, Cucchiara S, Civitelli F, et al. Colon capsule endoscopy compared with other modalities in the evaluation of pediatric Crohn's disease of the small bowel and colon. Gastrointestinal Endoscopy 2016;83:975-83.

[29] Petruzziello C, Calabrese E, Onali S, et al. Small bowel capsule endoscopy vs conventional techniques in patients with symptoms highly compatible with Crohn's disease. Journal of Crohn's and Colitis 2011;5:139-47.

[30] Petruzziello C, Onali S, Calabrese E, et al. Wireless capsule endoscopy and proximal small bowel lesions in Crohn's disease. World Journal of Gastroenterology 2010;16:3299-304.

[31] Kopylov U, Nemeth A, Koulaouzidis A, et al. Small bowel capsule endoscopy in the management of established Crohn's disease: clinical impact, safety, and correlation with inflammatory biomarkers. Inflammatory Bowel Diseases 2015;21:93-100

[32] Kopylov U, Ben-Horin S, Seidman EG, et al. Video capsule endoscopy of the small bowel for monitoring of Crohn's disease. Inflammatory Bowel Diseases 2015;21:2726-35

[33] Greener T, Shapiro R, Klang E, et al. Clinical outcomes of surgery versus endoscopic balloon dilation for stricturing Crohn's disease. Diseases of the Colon and Rectum 2015;58:1151-7.

[34] Rozendorn N, Klang E, Lahat A, et al. Prediction of patency capsule retention in known Crohn's disease patients by using magnetic resonance imaging. Gastrointestinal Endoscopy 2016;83:182-7.
[35] Pennazio M, Spada C, Eliakim R, et al. Small-bowel capsule endoscopy and device-assisted enteroscopy for diagnosis and treatment of small-bowel disorders: European Society of Gastrointestinal Endoscopy (ESGE). Endoscopy $2015 \cdot 47: 352-76$

[36] Nemeth A, Kopylov U, Koulaouzidis A, et al. Use of patency capsule in patients with established Crohn's disease. Endoscopy 2016;48:373-9.

[37] Kopylov U, Nemeth A, Cebrian A, et al. Symptomatic retention of the patency capsule: a multicenter real life case series. Endoscopy International Open 2016;4:E964-9.

[38] Amitai MM, Ben-Horin S, Eliakim R, et al. Magnetic resonance enterography in Crohn's disease: a guide to common imaging manifestations for the IBD physician. Journal of Crohn's and Colitis 2013;7:603-15.

[39] Cammarota T, Ribaldone DG, Resegotti A, et al. Role of bowel ultrasound as a predictor of surgical recurrence of Crohn's disease. Scandinavian Journal of Gastroenterology 2013;48:552-5.

[40] Danese S, Fiorino G, Fernandes C, et al. Catching the therapeutic window of opportunity in early Crohn's disease. Current Drug Targets 2014;15:1056-63.

[41] Fiorino G, Bonifacio C, Malesci A, et al. MRI in Crohn's disease-current and future clinical applications. Nature Reviews Gastroenterology \& Hepatology 2012;9:23-31.

[42] Wilkens R, Novak KL, Lebeuf-Taylor E, et al. Impact of intestinal ultrasound on classification and management of Crohn's disease patients with inconclusive colonoscopy. Canadian Journal of Gastroenterology and Hepatology 2016;2016, http://dx.doi.org/10.1155/2016/8745972, 8745972 (Epub 2016 Apr 19).

[43] Cheifetz AS, Kornbluth AA, Legnani P, et al. The risk of retention of the capsule endoscope in patients with known or suspected Crohn's disease. American Journal of Gastroenterology 2006;101:2218-22.

[44] Cohen SA, Gralnek IM, Ephrath H, et al. The use of a patency capsule in pediatric Crohn's disease: a prospective evaluation. Digestive Diseases and Sciences 2011;56:860-5.

[45] Dussault C, Gower-Rousseau C, Salleron J, et al. Small bowel capsule endoscopy for management of Crohn's disease: a retrospective tertiary care centre experience. Digestive and Liver Disease 2013;45:558-61.

[46] Herrerias JM, Leighton JA, Costamagna G, et al. Agile patency system eliminates risk of capsule retention in patients with known intestinal strictures who undergo capsule endoscopy. Gastrointestinal Endoscopy 2008;67:902-9.

[47] Höög CM, Bark L-Å, Arkani J, et al. Capsule retentions and incomplete capsule endoscopy examinations: an analysis of 2300 examinations. Gastroenterology Research and Practice 2012;2012, http://dx.doi.org/10.1155/2012/518718, 518718 (Epub 2011 Sep 29).

[48] Buisson A, Hordonneau C, Goutte M, et al. Diffusion-weighted magnetic resonance entero-colonography is highly effective to detect ileocolonic endoscopic ulcerations in crohn's disease. Alimentary Pharmacology Therapeutics 2015;42:452-60.

[49] Greenup A-J, Bressler B, Rosenfeld G. Medical imaging in small bowel Crohn's disease-computer tomography enterography, magnetic resonance enterography, and ultrasound. Inflammatory Bowel Diseases 2016;22:1246-61.

[50] Masselli G, Mastroiacovo I, De Marco E, et al. Current tecniques and new perpectives research of magnetic resonance enterography in pediatric Crohn's disease. World Journal of Radiology 2016;8:668-82.

[51] Knieling F, Waldner MJ. Light and sound-emerging imaging techniques for inflammatory bowel disease. World Journal of Gastroenterology 2016;22:5642-54.

[52] Gralnek IM, Defranchis R, Seidman E, et al. Development of a capsule endoscopy scoring index for small bowel mucosal inflammatory change. Alimentary Pharmacology Therapeutics 2008;27:146-54.

[53] Gal E, Geller A, Fraser G, et al. Assessment and validation of the new capsule endoscopy Crohn's disease activity index (CECDAI). Digestive Diseases and Sciences 2008;53:1933-7. 